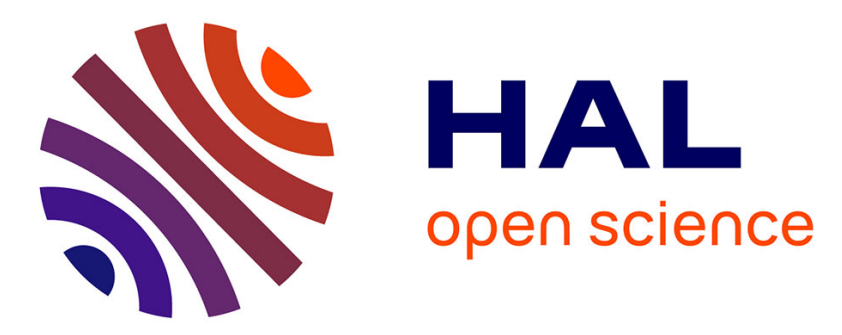

\title{
Durées de fluorescence des sels d'uranyle solides et de leurs solutions,
}

\author{
R. Delorme, F. Perrin
}

\section{To cite this version:}

R. Delorme, F. Perrin. Durées de fluorescence des sels d'uranyle solides et de leurs solutions,. J. Phys. Radium, 1929, 10 (5), pp.177-186. 10.1051/jphysrad:01929001005017700 . jpa-00205376

\section{HAL Id: jpa-00205376 https://hal.science/jpa-00205376}

Submitted on 1 Jan 1929

HAL is a multi-disciplinary open access archive for the deposit and dissemination of scientific research documents, whether they are published or not. The documents may come from teaching and research institutions in France or abroad, or from public or private research centers.
L'archive ouverte pluridisciplinaire HAL, est destinée au dépôt et à la diffusion de documents scientifiques de niveau recherche, publiés ou non, émanant des établissements d'enseignement et de recherche français ou étrangers, des laboratoires publics ou privés. 


\section{LE JOURNAL DE PHYSIQUE

\section{LE RADIUM}

\section{DURÉES DE FLUORESGENGE DES SELS D'URANYLE SOLIDES ET DE LEURS SOLUTIONS,}

par MM. R. DELORME et F. PERRIN.

Sommaire. - Les sels d'uranyle, ainsi que certaines de leurs solutions, excités par la lumière bleue, présentent une fluorescence verte durable que l'on peut observer par la méthode classique du phosphoroscope de Becquerel. La durée d'émission de cette fluorescence a été étudiée en adjoignant à un tel phosphoroscope un dispositif photométrique précis, qui permet de vórifier que la luminescence, après un éclairement instantané, décroît exponentiellement

$$
I=I_{0} \mathbf{e}^{-t_{i}}
$$

et de déterminer (à la température ordinaire et à celle de l'air liquide) la grandeur $\tau$ qui représente la vie moyenne des molécules dans l'état excité.

Ces mesures ont été faites :

$1^{\circ}$ pour les sels solides anciennement étudiés par Becquerel de façon peu précise.

$2^{\circ}$ pour les solutions dans l'acide sulfurique et l'acide métaphosphorique dont la fluorescence a une durée du même ordre que celle des cristaux, comme l'a découvert l'un de nous.

L'ensemble des résultats confirme l'hypothèse que la luminescence des sels d'uranyle est une fluorescence simple de longue durée, due a des transformations d'activation et de désactivation spontanée entre l'état normal et un état d'activation électronique presque métastable du groupe $\mathrm{U}^{2}$.

1. Dispositif expérimental. - Pour mesurer les durées d'émission des sels d'uranyle, j'ai employé le phosphoromètre représenté dans le schéma de la fig. 1. L'intensité de la lumière sortant du phosphoroscope doit être comparée à celle d'un étalon de même

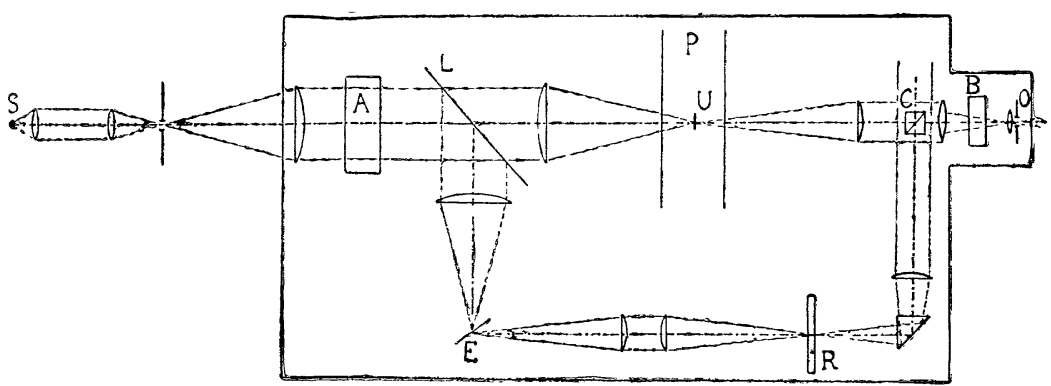

Fig. 1.

couleur et d'intensité proportionnelle à celle de la lumière excitatrice (qui peut n'être pas tout à fait constante). Ces conditions sont réalisées de la façon suivante :

Le faisceau lumineux donné par l'arc $S$ est rendu parallèle. Une glace sans tain $L$. inclinée à $45^{\circ}$ le divise en deux parties, ce qui assure une proportionnalité rigoureuse entre l'intensité totale de la lumière d'excitation qui pénètre dans le phosphoroscope et celle de la lumière qui excite l'écran de comparaison $E$.

Le faisceau réfléchi converge en $E$ sur la face antérieure d'un écran constitué, soit par de la poudre de sulfate d'uranyle serrée entre deux lamelles de verre, soit par une lame de

le journal de physique et le radium. - sérig vi. - t. x. - No 5 - mai 1929. 
verre d'urane. Cet écran, observé par sa face postérieure, constitue l'étalon de lumière. L'intensité du faisceau qui en émane peut être réduite dans un rapport connu au moyen d'une roue à secteurs d'ouverture réglable, animée, par un petit moteur électrique, d'une rotation rapide.

Le faisceau transmis converge sur le phosphoroscope $P$. Ce dernier est un phosphoroscope à disques du type $\mathrm{E}$. Becquerel. Les deux disques, de $15 \mathrm{~cm}$ de diamètre, portent chacun 16 entailles en forme de secteurs de $4,5^{\circ}$ disposées symétriquement à la périphérie. Ils sont enfermés dans des carters percés de fenêtres de même ouverture limitées verticalement par des arcs de cercle. Ils sont montés rigidement sur un axe commun de façon que la bissectrice d'une entaille de l'un des disques soit parallèle à la bissectrice d'un secteur plein situé entre deux entailles consécutives de l'autre disque. Toutes les entailles d'un des disques sont ainsi disposées en chicane par rapport aux entailles de l'autre disque. La distance interne entre les deux carters est d'environ $5 \mathrm{~cm}$, ce qui permet d'introduire entre eux un petit vase de Dewar en quartz transparent non argenté pour les mesures dans l'air liquide ('). Le système axe-disques est entraîné par un jet d'air comprimé frappant une petite roue à aubes montée sur l'axe. Enfin l'axe porte à l'une de ses extrémités une vis sans fin sur laquelle s'engrène une roue tangente qui établit un contact électrique tous les cinq cents tours. Ces contacts s'inscrivent, ainsi que les battements d'un chronomètre donnant le cinquième de seconde, sur une bande de papier enfumée animée d'une vitesse uniforme. La vitesse de rotation des disques est ainsi continuellement enregistrée Elle peut atteindre deux cents tours par seconde.

Le faisceau de fluorescence émis par la substance étudiée $U$ émerge du phosphoroscope, est rendu parallèle et tombe en $C$ sur un cube de Lummer, perpendiculairement au faisceau de comparaison. Enfin, grâce à un système de lentilles, on superpose sur un petit orifice $O$ l'image de l'étalon $E$ et de la substance $U$ étudiée. L'oeil, placé en $O$, perçoit les deux plages du cube de Lummer. L'éclairement est uniforme sur chacune des plages.

La substance étudiée $U$ est prise, dans le cas des solides, sous la forme de poudre serrée entre deux lamelles de quartz, comme l'étalon; dans le cas des solutions, elle remplit un petit tube en quartz fondu transparent; tubes et lamelles sont fixés rigidement au bâti général.

La lumière excitatrice est filtrée par un écran bleu (cuivre ammoniacal), et la fluorescence est observée à travers un écran complémentaire jaune (chromate de potassium), ce qui élimine toute lumière diffusée.

2. Mesures. - On donne au secteur variable de la roue $\mathrm{R}$ une ouverture angulaire connue et on la fait tourner à une vitesse suffisante pour que le faisceau qui la traverse donne une sensation visuelle continue (une quinzaine de tours par seconde). La plage de comparaison du cube de Lummer a ainsi une brillance constante $I$, proportionnelle à l'angle d'ouverlure du secteur variable. On lance alors progressivement le phosphoroscope. L'intensité sur la deuxième plage du cube croît, à partir de zéro, avec la vitesse de rotation des disques. Un manipulateur électrique permet de noter sur l'enregistreur à noir de fumée l'instant où les deux plages passent par la même intensité. La vitesse continuant à croitre légèrement, la plage correspondant à la substance étudiée devient plus lumineuse que la plage de comparaison. On refait la détermination en sens inverse en faisant décroître lentement la vitesse, et notant à nouveau sur l'enregistreur l'instant où les deux plages passent par l'égalité.

La vitesse de rotation correspondant à l'intensité $I$ est prise égale à la moyenne des deux vitesses marquées sur l'enregistreur.

On recommence cinq ou six fois une telle opération en donnant au secteur variable une série d'ouvertures différentes. On obtient ainsi, pour la substance étudiée, la variation de l'intensité lumineuse moyenne du faisceau qui émerge du phosphoroscope en fonction de la vitesse de rotation des disques (caractérisée par le nombre $N$ de tours par seconde, ou par la période de rotation $T$ ).

(1) Le verre', ayant une légère fluorescence, n'a pas été employé à l'intérieur du phosphoroscope. 
3. Interprétation des résultats. - Il pourrait sembler que le temps qui s'écoule entre un éclairement et une observation consécutive étant inversement proportionnel à la vitesse, il suffise de construire la courbe $I$ en fonction de $T$ pour avoir la loi de décroissance exponentielle. En réalité la courbe ainsi obtenue n'est que grossièrement exponentielle dans sa partie médiane et conduit pour $\tau$ à des valeurs tout à fait erronées $\left({ }^{2}\right)$. Il faut en effet d'une part tenir compte de l'ouverture progressive des fenêtres d'éclairement et d'observation, et d'autre part totaliser les émissions dues aux excitations successives.

L'interprétation correcte des résultats' exige ainsi le calcul suivant :

Appelons q l'angle d'ouverture des entailles, s'il y a $p$ entailles sur chaque disque, nous poserons $\Phi=\frac{2 \pi}{2 p} ; \Phi$ est l'angle des bissectrices de deux entailles successives prises sur un disque et sur l'autre. Pour une vitesse angulaire permanente donnée $\omega$ nous pouvons poser, en introduisant les temps à la place des angles :

$$
\varphi=\omega \theta \quad \text { et } \quad \Phi=\omega \Theta .
$$

Prenons comme origine des temps l'instant où, par le passage d'une entaille, lá fenêtre par où pénètre le faisceau excitateur commence à s'ouvrir et étudions la variation de l'intensité de la lumière d'excitation pendant le passage de cette entaille. Si le faisceau excitateur couvre entièrement et uniformément la fenêtre d'entrée du phosphoroscope, ce qui estipratiquement réalisé, l'intensité $f(\omega t)$ qui tombe sur la substance à chaque inslant $t$ est, pendant le temps $\theta$ à partir de l'origine des temps, proportionnelle à l'angle $\omega t$ dont a tourné le disque, et de l'instant $\theta$ à l'instant $2 \theta$ proportionnelle à l'angle $(2 \theta-t) \omega$.

Nous admettrons que conformément à la théorie des fluorescences simples, la luminescence des sels d'uranyle, due à une excitation instantanée, décroît exponentiellement à partir de l'instant de cette excitation, et que des excitations successives agissent indépendamment les unes des autres, les émissions correspondantes s'ajoutant simplement. Ces hypothèses seront à posteriori vérifiées par la concordance de la loi théorique obtenue avec la loi expérimentale de variation de la luminosité en fonction de la vitesse de rotation.

L'intensité de la fluorescence élémentaire due à l'excitation reçue à l'instant $t$, pendant une durée infiniment petite $\mathrm{d} t$, est d'une part proportionnelle à l'intensité totale $f(\omega t) \mathrm{d} t$ de cette excitation instantanée, et d'autre part décroît exponentiellement à partir de l'instant $t$ où elle s'est produite. A un instant ultérieur $t^{\prime}$, elle sera donc proportionnelle à

$$
f(\omega t) \mathrm{d} t \cdot \mathrm{e}^{-a\left(t^{\prime}-t\right)} \text {. }
$$

On voit alors assez facilement que l'intensité de fluorescence due à toutes les excitations élémentaires étendues à toutes les entailles successives qui ont passé devant la fenêtre d'entrée est, à un instant où la fenêtre d'observation commence exactement à s'ouvrir, donnée, à un facteur constant près, par l'expression :

$$
\varepsilon_{\Phi}=\sum_{n=0}^{\infty}\left[\int_{0}^{\theta} k \omega t \cdot \mathrm{e}^{-a[(2 n+1) \Theta-t]} \mathrm{d} t+\int_{\theta}^{2 \theta} k \omega(2 \theta-t) \cdot \mathrm{e}^{-a[(2 n+1) \Theta-t]} \mathrm{d} t\right]
$$

qui se réduit, tous calculs faits, à

$$
\xi_{\Phi}=k \omega \frac{\mathbf{e}^{-a \Theta}}{1-\mathbf{e}^{-2 a \Theta}} \frac{1}{a^{2}}\left(1-\mathbf{e}^{a \theta}\right)^{2} .
$$

A partir de cet instant, tandis que l'intensité de fluorescence continue à décroitre exponentiellement, l'intensité vraie qui sort du phosphoroscope est proportionnelle à l'angle d'ouverture de la fenètre d'observation.

Un raisonnement tout à fait analogue au précédent montre que la somme des inten-

(2) Les vies moyennes données par E. Becquerel, qui se servait de cette courhe ałec une petite correction, sont presque deux fois trop grandes. 
sités élémentaires émises pendant le passage d'une des entailles devant la fenêtre d'observation est de la forme

$$
\varepsilon_{\varphi}=\varepsilon_{\Phi}\left[\int_{0}^{\theta} \omega t \mathrm{e}^{-a t} \mathrm{~d} t+\int_{\theta}^{2 \theta} \omega(2 \theta-t) 6^{-a t} \mathrm{~d} t\right] .
$$

Enfin, pour avoir l'intensité moyenne observée $I$, il faut diviser $\varepsilon_{\curlyvee}$ par $2 \Theta$.

En transformant l'expression de $l$ et introduisant les angles $p$ et $\Phi$ on troure

$$
l=k \frac{1}{a} \frac{1}{u^{3}} \frac{1}{\Phi} \frac{(\operatorname{ch} u \varphi-1)^{2}}{\operatorname{sh} u \Phi} \quad \text { avec } \quad u=\frac{a}{\omega} .
$$

On voit que si $u$ tend vers 0 , c'est-à-dire si la vitesse de rotation des disques devient infinie, $I$ tend vers un maximum

$$
I_{m}=\frac{k}{4 a} \frac{\varphi^{4}}{\Phi^{\dddot{y}}}
$$

ce qui est conforme à l'expérience. En mettant en évidence cette intensité maximum, on peut écrire :

$$
I=I_{m} \frac{4 \Phi}{\varphi^{4}} \frac{1}{u^{3}} \frac{(\operatorname{ch} u \varphi-1)^{2}}{\operatorname{sh} u \varphi}=I_{m} \frac{2 \Phi}{\varphi}\left(\frac{\omega}{a_{\varphi}}\right)^{3} \frac{\mathbf{e}^{-u(\Phi-2 \vartheta)}}{1-\mathbf{e}^{-2 u \Phi}}\left(1-\mathbf{e}^{-u_{\rho}}\right)^{4} .
$$

Cette formule est générale pour un phosphoroscope dont les disques portent des

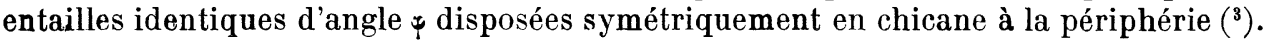

Dans le cas des disques employés ici :

$$
\Phi=\frac{\pi}{16}, \quad \varphi=\frac{\pi}{40}
$$

nous avons d'autre part $\omega=2 \pi / T, T$ étant la période de rotation des disques, et $a=1 / \tau$, en désignant par $\tau$ la durée moyenne d'émission. Si l'on porte ces valeurs dans la formule précédente, on a :

$$
I=I_{m} \breve{5}\left(\frac{\partial}{16 \tau}\right)^{3} \frac{\mathrm{e}^{-\frac{1}{10} \frac{T}{16 \tau}}}{1-\mathrm{e}^{-\frac{T}{16 \tau}}}\left(1-\mathrm{e}^{-\frac{1}{5} \frac{T}{16 \tau}}\right)^{4}
$$

On peut construire d'après cette formule la courbe théorique donnant $\log \left(I_{m} / I\right)$ en fonction de $\log (T / 16 \tau)$. Si la loi de décroissance élémentaire de la luminescence est bien exponentielle, la courbe expérimentale donnant $\log (\boldsymbol{A} / \boldsymbol{I})$ ( $A$ étant une intensité lumineuse arbitraire) en fonction de $\log (T / 10 \tau)$ doit se superposer à la précédente par une translation dont la composante suivant l'axe des abscisses donnera log $\tau$.

En fait, la superposition a toujours été bonne dans toute la partie médiane de la courbe expérimentale, ce qui justifie, a posteriori, les hypothèses faites au cours du calcul et vérifie notamment la loi de décroissance exponentielle admise $\left(^{4}\right)$.

(3) Par un calcul analogue, dans lequel il tenait compte des exsitations par les entailles successives, mais non de l'ouverture progressive des fenêtres, Wiedemann avait obtenu une formule un peu différente qui lui avait permis de corriger d'une façon déjà importante les nombres de E. Becquerel [Wiedemann Ann., t. 34 (1888), p. 446].

(\$) Nichols, cherchant à vérifier une idée théorique, avait cru pouvoir déduire d'une étude expérimentale de la luminescence des sels d'uranyle une autre loi de décroissance : la courbe représentant, en fonction du temps, la racine carrée de l'intensité émise après une excitation, aurait été constituée par une suite de plusieurs segments de droite formant des angles brusques. Mais S.-J. Wawilow et W.-L. Lewschin ont montré que les mesuros de Nichols étaient bien mieux interprétées par une loi simple de décroissance exponentiolle que par ces lois complexes et d'ailleurs surprenantes. 
La coïncidence est souvent moins bonne aux très faibles et aux très grandes vitesses. Je vois à cola deux causes secondaires : l'enregistreur ne permet pas, dans ces conditions, des mesures très précises. D'autre part, l'égalité d'éclairement est, dans ce cas, moins facile à apprécier : aux faibles vitesses, la luminosité est peu visible; aux très grandes vitesses, il est difficile d'éviter une petite différence de teinte entre les plages alors très brillantes.

La figure 2 représente une de nos déterminations.

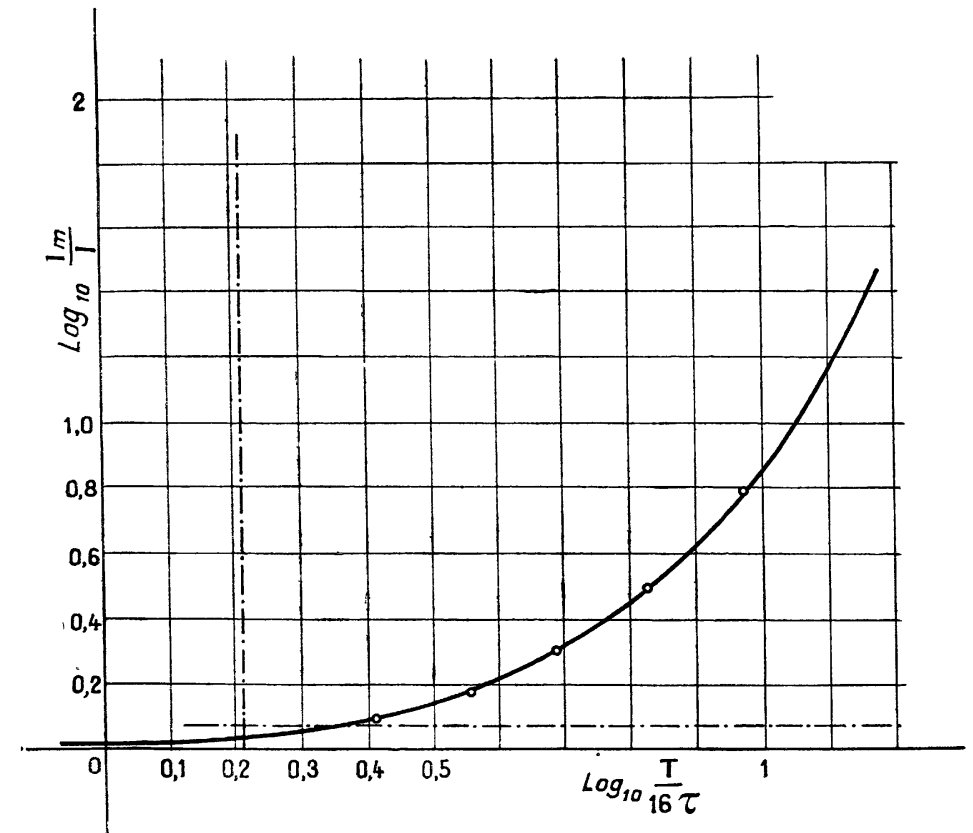

Fig. 2. - Détermination de la vie moyenne du chlorure d'uranyle à la température ordinaire.

Axes et courbe théoriques.

Axes de la courbe expérimentale, dont les points seuls sonţ indiqués.

Unité de temps pour la courbe expérimentale : $U_{t}=2,5 \times 10^{-4} \mathrm{~s}$.

La superposition des courbes donne : $\log U_{t}-\log \tau=0,21, \tau=1,54 \times 10^{-4}$.

4. Valeurs de la vie moyenne pour les sels solides. Influence de la température. - Les premiers résultats obtenus par cette méthode ont fait l'objet d'une note aux Comptes rendus de l'Académie $\left(^{5}\right)$.

Ils sont reproduits dans le Tableau I avec des mesures nouvelles, et quelques déterminations faites à la température de l'air liquide.

L'erreur probable est de $\stackrel{3}{\text { p. } 100 .}$

Ces valeurs sont très différentes de celles données par $\mathbf{E}$. Becquerel qui, comme nous l'avons dit, appliquait à ses mesures une méthode de calcul tout à fait insuffisante. Les valeurs déduites de ces mêmes mesures par Wiedemann se rapprochent des nôtres, mais sont certainement moins précises.

Nos valeurs sont, au contraire, dans l'ensemble, en très bon accord avec celles obtenues par W.-L. Lewschin et S.-J. Wavilow, au moyen de leur phosphoromètre à miroir tournant $\left(^{6}\right)$. (Ces physiciens ont d'ailleurs montré que les mesures de Nichols convenablement interprétées conduisaient à des valeurs voisines des leurs).

(5) F. Perrin et R. Delorme, C. R., t. 186 (février 1928), p. 428.

(') Zts.f. Phys., t. 48 (mars 1928) p. 397. 
Tableav I.

\begin{tabular}{|c|c|c|}
\hline \multirow{2}{*}{ - SRIS D'URANYLB SOLIDES } & \multicolumn{2}{|c|}{$\tau 10^{4}$ (en secondes) } \\
\hline & à $20^{\circ} \mathrm{C}$ & $\grave{a}-180^{\circ} \mathrm{G}$ \\
\hline Sulfate cristallisé. & $2, \check{5}$ & $3, \breve{5}$ \\
\hline Nitrate cristallisé à $6 \mathrm{H}_{2} \mathrm{O} \ldots \ldots \ldots \ldots \ldots \ldots$ & 6,1 & 8 \\
\hline Chlorure $\left(^{7}\right) \ldots \ldots \ldots \ldots \ldots \ldots \ldots \ldots$ & 1,34 & 3,6 \\
\hline 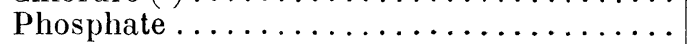 & 0,5 & \\
\hline Acétate $\ldots \ldots \ldots \ldots \ldots \ldots \ldots$ & 1,34 & \\
\hline$\left(\mathrm{SO}_{4}\right)_{2} \mathrm{UO}_{2} \mathrm{~K}_{2}, 2 \mathrm{H}_{2} \mathrm{O}\left({ }^{8}\right) \ldots \ldots \ldots \ldots$ & 1,66 & \\
\hline Verres d'urane ( 2 provenances).... & $\begin{array}{l}3,2 \\
2,9\end{array}$ & \\
\hline
\end{tabular}

Les déterminations faites à la température de l'air liquide $\left(-180^{\circ} \mathrm{C}\right)$ montrent que les durées moyennes d'émission sont un peu plus grandes qu'à la température ordinaire, mais cependant du même ordre de grandeur, tandis que pour les phosphorescences la durée d'émission augmente indéfiniment quand on abaisse la température. La luminescence des sels d'uranyle se comporte donc à ce point de vue comme une fluorescence. L'augmentation, peu importante, relativement, de la durée d'émission est probablement corrélative d'une augmentation du rendement lumineux ; elle est en effet la plus forte pour le chlorure d'uranyle $\left({ }^{9}\right)$ dont la fluorescence augmente le plus par abaissement de température.

Enfin il faut remarquer que pour l'ensemble des sels étudiés la durée moyenne d'émission n'est pas en relation directe avec le pouvoir fluorescent. Par exemple, le sulfate, plus fluorescent que le nitrate, a une durée d'émission plus de moitié moindre. On peut cependant affirmer que tous les composés peu fluorescents ont des durées d'émission relativement petites.

๖. Etude des solutions de sulfate d'uranyle dans l'acide sulfurique. - Les solutions des sels d'uranyle sont, en général, beaucoup moins fluorescentes que les sels cristallisés, et leurs luminescences faibles, invisibles dans les phosphoroscopes mécaniques, étaient considérées comme considérablement plus brèves et même d'une nature différente de celles des solides (fluorescence instantanée et non plus phosphorescence brève). Parmi ces solutions, les plus fluorescentes sont celles du sulfate d'uranyle en milieu acide; et si l'on dissout ce sel dans l'acide sulfurique concentré pur, on obtient une solution dont la fluorescence est très brillante et comparable à celle des sels solides. En étudiant cette solution, dont la viscosité est seulement vingt fois celle de l'eau, au moyen d'un phosphoroscope ordinaire de E. Becquerel, l'un de nous $\left({ }^{10}\right)$ constata aisément que sa luminescence avait une durée du même ordre de grandeur que pour les sels cristallisés, et seulement environ quatre fois plus brève que celle des cristaux de nitrate d'uranyle ( $\left.{ }^{11}\right)$.

(7) Le chlorure d'uranyle a été préparé en dissolvant $\mathrm{CO}_{3}$ dans $\mathrm{HCl}$ et évaporant dans le vide la solution obtenue. $\mathrm{UO}_{3}$ était obtenu en chauffant le nitrate au bain d'huile à $250^{\circ} \mathrm{C}$ jusqu'à ce qu'il ne se dégage plus de vapeurs nitreuses. Le chlorure, très déliquescent, était enfermé dès sa sortie de la cloche à vide entre les lamelles de quartz que je collais à la cire.

(8) Ce sel a été obligeamment prêté par M. A. Urbain.

( $\left.{ }^{9}\right)$ Comme l'avait déià remarqué qualitativement J. Becquerel [C. R., t. 1 (1911), p. 511].

(10) Francis Perrin, C. R., t. 182 (1926), p. 929.

(11) La possibilité d’observer cette luminescence au 'moyen du phosphoroscope ordinaire de Becquerel (4 entailles par disque, 100 tours par seconde) a été contestée par S.-J. Wawilow et W.-L. Lewschin. L'expérience est pourtant facile et la luminescence observée aux grandes vitesses de rotation, très brillante. Il suffit d'ailleurs de remarquer que la durée moyenne d'émission de ces solutions est seulement moitié de celle du verre d'urane, dont l'observation au moyen du phosphoroscope de Becquerel est classiquement tout à fait aisée, sans qu'il soit nécessaire de donner aux disques une grande vitesse de rotation; en les faisant tourner simplement deux fois plus vite, ce qui est facile, on observe de même très brillamment l'émission de la solution sulfurique de sulfate. 
La fluorescence de ces solutions est très sensible à des traces d'impureté, notamment acides halogénés ou substances organiques; pour éviter ces impuretés, le plus simple est d'oxyder la solution en la faisant bouillir après addition d'un peu d'acide nitrique fumant, ou, ce qui revient au même, de préparer la solution sulfurique de sulfate, en dissolvant dans l'acide sulfurique concentré $\left(66^{\circ} \mathrm{B}\right)$ du nitrate d'uranyle et en chassant l'acide nitrique libéré par ébullition. Nos solutions ont été préparées par cette dernière méthode.

Le pouvoir fluorescent des solutions sulfuriques de sulfate d'uranyle dépend en outre de la concentration et surtout de la température; et ces facteurs modifient dans le méme sens la durée moyenne d'émission $\tau$. On peut d'ailleurs s'attendre à une variation proportionnelle de ces deux grandeurs, conformément à la relation théorique

$$
\tau=\rho \tau_{0},
$$

où $\rho$ désigne le rendement lumineux (en nombre de quanta) de la fluorescence, et $\tau_{0}$, la durée moyenne maximum d'émission, correspondant au cas d'un rendement égal à l'unité $\left({ }^{12}\right)$, pour le corps considéré.

A la température ordinaire $\left(17^{\circ}\right)$, la plus grande durée d'émission s'obtient pour les solutions étendues dans l'acide sulfurique pur, pour lesquelles les mesures m'ont donné

$$
\tau=1,4 \cdot 10^{-i} \text { seconde. }
$$

Quand la concentration augmente, quand la température s'élève ou quand on additionne d'eau la solution, la durée moyenne d'émission diminue; les résultats des mesures sont rassemblés dans le tableau suivant :

TABLEAU II.

\begin{tabular}{|c|ccc|}
\hline concentration $C$ en $\left(\mathrm{SO}_{4} \mathrm{LO}_{2}\right)$ & \multicolumn{3}{|c}{$\tau \cdot 10^{2}$ (en secondes) } \\
$\mathrm{g}: \mathrm{cm}^{3}$ & $1^{0^{2}}$ & $17^{\circ}$ & $28^{\circ}$ \\
\hline $7,2.10^{-3}$ & 2,0 & 1,4 & 1,25 \\
$7,2.10^{-2}$ & & 1,0 & \\
$7,2.10^{-1}$ & & 0,9 \\
\hline
\end{tabular}

Enfin pour une solution contenant 30 pour 100 d'eau, j'ai trouvé $\tau=0,55.10^{-4} \mathrm{~s}$.

Ces valeurs, déjà publiées $\left({ }^{13}\right)$, et celles de W.-L. Lewschin et S.-J. Wawilow (14) sont dans l'ensemble en bon accord (sauf dans le cas des solutions très concentrées, d'ailleurs assez facilement altérables, pour lesquelles la valeur donnée ici est presque double). Ces auteurs ont d'autre part vérifié de façon satisfaisante la proportionnalité prévue du pouvoir fluorescent et de la durée moyenne d'émission, quand la concentration, la température on la teneur en eau varient.

Nous avons fait cette vérification dans le cas de la diminution de fluorescence par addition à la solution d'une très petite quantité d'acide chlorhydrique. La sensibilité des solutions sulfuriques de sulfate d'uranyle aux inhibiteurs de fluorescence est telle qu'il suffit d'un trente-millième d'acide chlorhydrique pouf réduire de moitié la durée moyenne d'émission et le pouvoir fluorescent $\Phi$. De façon précise, les mesures ont donné pour deux

(12) F. Pbrrin, C. R., t. 182 (1926), p. 219 et J. Phys., t. 7 (1926), p. 390.

(13) F. Perrin et R. Delorme, loc. cit.

(14) loc. cit. 
solutions ne différant que par la présence dans l'une d'elles d'un trente-millième d'acide chlorhydrique (en grammes de HCl par centimètre cube) :

$$
\frac{\tau_{1}}{\tau_{2}}=2,08 \quad \text { et } \quad \frac{\Phi_{1}}{\Phi_{2}}=2,04 \text {. }
$$

Le rapport des pouvoirs fluorescents a été déduit des mesures phosphorométriques elles-mêmes; pour deux telles solutions, ayant la même concentration en sulfate d'uranyle et placées dans le même récipient, ce rapport est en effet égal au rapport des intensités maximum $I_{m}$, donné par la différence des composantes suivant l'axe des ordonnées des translations qui permettent d'amener en coïncidence les deux courbes expérimentales et la courbe théorique sur le graphique logarithmique.

6. Etude des solutions de métaphosphate d'uranyle. Influence de la viscosité. - La viscosité est visiblement un facteur qui agit de façon importante sur le pouvoir fluorescent et la durée d'émission des solutions de sel d'uranyle : Toutes choses égales d'ailleurs, le rendement de fluorescence et la durée moyenne d'émission augmentent avec la viscosité. Mais il est difficile de faire varier la viscosité seule et sans modifier quelque autre facteur d'action (température, concentration.....) $\left.{ }^{15}\right)$. Nous avons espéré y réussir le mieux en étudiant les solutions de métaphosphate d'uranyle dans l'acide métaphosphorique plus ou moins étendu d'eau, dont les viscosités s'étendent sur un grand intervalle depuis la viscosité de l'eau jusqu'à des viscosités pratiquement infinies (état vitreux très dur). Malheureusement, la manipulation de ces solutions exige qu'on les chauffe assez fréquemment, ce qui produit une transformation partielle de l'acide méta on acide orthophosphorique, et cette modification chimique peut influencer aussi la fluorescence.

Les viscosités ont été mesurées, assez grossièrement d'ailleurs, en comparant des temps d'écoulement (à 18) à travers une série de tubes calibrés.

Le tableau III donne les durées moyennes d'émission en fonction de la viscosité pour une série de solutions de métaphosphate d'uranyle dans l'acide métaphosphorique pur ou mélangé d'eau :

TableaU III.

\begin{tabular}{|c|c|}
\hline viscosité (cgs) & $\tau .10^{4}$ (en secondes) \\
\hline $\begin{array}{l}0,02 \\
1,4 \\
100 \\
20000 \\
\\
\quad ? \text { (pâte) } \\
\quad \text { ? (acide pur vitreux) }\end{array}$ & $\begin{array}{l}0,9 \\
1,0 \\
2,3 \\
3,8 \\
5,6 \\
7,0\end{array}$ \\
\hline
\end{tabular}

7. Interprétation des résultats. - On sait que la différence essentielle entre fluorescence et phosphorescence ne réside pas dans une plus ou moins grande persistance d'émission, car à ce point de vue une suite à peu près continue d'intermédiaires relie les fluorescences les plus brèves aux phosphorescences les plus longues.

Une fluorescence est essentiellement associée à un simple passage des molécules ayant absorbé la lumière incidente, par un état excité à partir duquel l'émission se produit spontanément par suite d'une instabilité interne; et si cet état n'est pas directement atteint au moment de l'absorption, les transformations intermédiaires doivent se produire presque instantanément et n'emprunter aucune énergie au milieu (amortissement rapide d'un mouvement d'oscillation produit en même temps que l'excitation électronique). La

(15) Cf. W.-L. Lewschin et S.-J. Wawilow, loc. cit. 
durée moyenne d'une fluorescence ainsi définie est donc simplement la vie moyenne $\tau$ des molécules dans un état excité susceptible de transformation spontanée par émission ; elle est par suite au plus égale à l'inverse $\tau_{0} d u$ coefficient de probabilité de cette émission spontanée. D’une façon précise on a, p désignant le rendement lumineux,

$$
\tau=\rho \tau_{0}
$$

et cette relation permet de caractériser expérimentalement une vraie fluorescence.

$\mathrm{Si}$, au contraire, les molécules excitées passent, entre l'absorption et l'émission, par un état intermédiaire stable ou métastable, et ne peuvent plus alors atteindre l'état d'émission sans recevoir du milieu un certain incrément d'énergie, la luminescence est une phosphorescence, caractérisée par l'augmentation indéfinie de la durée d'émission aux basses températures.

Enfin, du point de vue ondulatoire, la durée d'une fluorescence représente la durée même de l'émission élémentaire, la constante d'amortissement des trains d'ondes, tandis que la durée d'une phosphorescence n'a, à ce point de vue, aucune signification fondamentale.

D'après ces critériums, la luminescence des sels d'uranyle est une fluorescence véritable, malgré sa persistance plus de dix mille fois plus grande que celles des fluorescences usuelles $\left(\tau=0,4.10^{-8} \mathrm{~s}\right.$ pour les solutions de fluorescéine) $\left({ }^{16}\right)$. La durée moyenne de cette luminescence doit être égale à la vie moyenne du radical $\left(\mathrm{UO}_{2}\right)$ dans un état d'activation électronique susceptible d'émission spontanée, et cette durée, ou plis exactement son quotient par le rendement lumineux correspondant, détermine l'inverse du coefficient de probabilité de cette émission spontanée. Du point de vue ondulatoire, la durée moyenne d'émission observée représente la période d'amortissement des trains d'ondes émis, qui s'étendraient par suite dans ce cas sur une centaine de kilomètres de longueur.

Un amortissement aussi faible aurait été tout à fait inexplicable par la théorie électromagnétique classique qui prévoyait une constante de temps d'amortissement de l'ordre de $10^{-8} \mathrm{~s}$ (pour une émission dans le spectre visible due à un électron oscillant). Il s'interprète au contraire aisément en mécanique ondulatoire. La vie moyenne maximum $\tau_{0}$ dans l'état excité est en effet déterminée par la condition

$$
\varepsilon \tau_{0}=h \nu \text {, }
$$

$\varepsilon$ représentant le pouvoir émissif, d'après la théorie classique $\left({ }^{17}\right)$, d'un oscillateur de fréquence $v$ et d'amplitude $q$

$$
\varepsilon=\frac{16 \pi^{4}}{3} n \frac{e^{2}}{c^{3}} v^{\prime} q^{2}
$$

( $n$, indice du milieu ; $c$, vitesse de la lumière ; $e$, charge de l'électron en U. E. S.). Mais cette amplitude, au lieu d'être reliée à l'énergie disponible $W=h$ v por la formule $W=2 \pi^{2} m \nu^{2} q^{2}$ valable pour une particule oscillante de masse $m$, en est indépendante. Ses composantes suivant les axes doivent être calculées par les formules

$$
q_{x}=\int x \psi_{1} \psi_{2}^{*} \mathrm{~d} x \mathrm{~d} y \mathrm{~d} z, \ldots, \ldots
$$

$\psi_{1}$ et $\psi_{2}$ représentant les fonctions de Schrödinger correspondant à l'état normal et à l'état excité considéré. Or on sail que l'amplitude ainsi associée à deux états quantiques peut être nulle, la transition entre ces états étant alors «interdite 》 par émission (état excité métastable). Mais elle peut aussi ètre, dans certains cas, très petite sans être nulle, quelle que soit d'ailleurs la grandeur de l'énergie disponible; l'émission sera alors possible, mais se produira en un temps très long, avec un amortissement très faible.

(16) F. Prrais, J. Phys., t. 7 (1926), p. 390

(17) Une théorie purement quantique de l'émission, développée par Dirac, conduit au même résultat que l'application faite ici du principe de correspondance. 
Pour qu'il en soit ainsi, il faut que les fonctions qui figurent dans les intégrales précédentes soient presque orthogonales. Et par suite, dans ce cas, une très faible perturbation des fonctions fondamentales $\psi$, résultant d'une petite modification des conditions extérieures, pourra modifier beaucoup la valeur relative de l'amplitude $q$, donc de la vie moyenne $\tau_{0}$. Autrement dit lorsqu'un état excité est presque métastable, la durée d'émission, beaucoup plus longue que celle prévue par la théorie classique, doit être sensible à de légères modifications des conditions extérieures, de structure moléculaire par exemple.

Ceci permet de comprendre que les divers sels d'uranyle aient des durées d'émission assez différentes, indépendamment des rendements lumineux, car le groupement $\left(\mathbf{U} \mathbf{O}_{2}\right)$ doit subir de petites perturbations, variables suivant la molécule dans laquelle il se trouve engagé, et qui peuvent influer notablement sur les conditions de quasi-orthogonalité, nécessaires pour expliquer les grandes durées d'émission.

Ainsi la durée relativement très grande de la luminescence des sels d'uranyle n'a rien d'inadmissible pour une fluorescence véritable; c'est la plus longue actuellement connue, mais des durées presque aussi grandes ont été observées même dans le domaine très simple des résonances optiques : par exemple, la deuxième raie de résonance du zinc correspond à une vie moyenne de $10^{-3} \mathrm{~s}$, près de cinq mille fois plus grande que la durée prévue pour sa fréquence par l'électromagnétisme classique $\left({ }^{18}\right)$.

8. Conclusions. - 1. La décroissance exponentielle de la luminescence des sels d'uranyle solides, ou en solution, après une excitation, a été vérifiée.

2. La durée moyenne d'émission - a été déterminée, avec une précision de 4 à 5 pour 100 pour un certain nombre de sels cristallisés. Les valeurs obtenues, très différentes des valeurs peu précises de E. Becquerel, sont en bon accord avec celles, récentes, de W.-L. Lewschin et S.-J. Wawilow. Elles varient, pour les sels les plus fluorescents, de 2 à $7.10^{-4} \mathrm{~s}$, sans relation directe avec le pouvoir fluorescent.

3. Les durées moyennes d'émission des sels solides très fluorescents (sulfate, nitrate) augmentent, mais relativement assez peu, quand on abaisse la température à - $180^{\circ}$.

4. Les solutions diluées de sulfate d'uranyle dans l'acide sulfurique pur (viscosité 0,2 ) présentent une belle fluorescence dont la durée moyenne d'émission est du même ordre de grandeur que celle des sels cristallisés (F. Perrin). La valeur trouvée pour cette durée

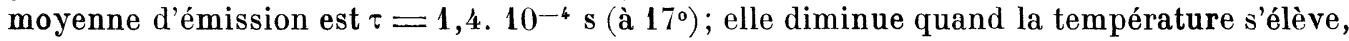
quand la concentration augmente, ou quand on ajoute de l'eau. Elle diminue également quand on introduit dans la solution des traces de certains corps : un trente-millième d'acide chlorhydrique réduit à la fois de moitié la durée moyenne d'émission et le pouvoir fluorescent.

๖. Bien que des solutions, même très fluides, puissent avoir des durées d'émission comparables à celles des composés solides, la viscosité agit cependant de façon importante sur les durées d'émission, (mais seulement pour autant qu'elle influe sur le pouvoir fluorescent).

Par exemple, dans le cas des solutions de métaphosphate d'uranyle dans l'acide métaphosphorique plus ou moins dilué, $\tau$ augmente depuis la valeur $0,9.10^{-4} \mathrm{~s}$ pour une solution de viscosité seulement double de celle de l'eau jusqu'à $7.10^{-4} \mathrm{~s}$ pour une solution vitreuse très dure.

6. L'ensemble de ces résultats, joint à ce qu'on sait déjà sur la luminescence des sels d'uranyle, vient fortement à l'appui de l'hypothèse que cette luminescence est une fluorescence simple (c'est-à-dire une activation électronique suivie d'une désactivation spontanée), dont la durée, environ cent mille fois plus grande que pour les fluorescences des matières colorantes, est remarquablement longue.

Ce travail a été fait au laboratoire de Chimie physique de M. le Professeur Jean Perrin.

(18) P. Solrillgt, C. R , t. 187 (1928), p. т23.

Manuserit reçu le 23 novembro 1928. 